\title{
Internet Addiction and its relationship with Emotional Intelligence and Perceived Stress experienced by Young Adults
}

\author{
Hemali Sanghvi ${ }^{1}$, Dr. Upagya Rai $^{2}$
}

\section{ABSTRACT}

The aim of this research was to assess Internet addiction and its relationship with Emotional Intelligence and Perceived stress among Young Adults in Bangalore. The study was conducted on a sample of 30 students from various universities across the city of Bangalore. Three scales were used for the purpose of the study, namely the Internet Addiction Test, the Emotional Intelligence Scale and the Perceived Stress Scale. Pearson Product Moment Correlation was employed to study the correation between Internet Addiction and emotional intelligence and between Internet addiction and perceived stress. The results of the study indicated that there is no significant correlation between Internet addiction and emotional intelligence and there is no significant correlation between Internet addiction and perceived stress.

Keywords: Internet Addiction, Emotional Intelligence, Perceived Stress

Internet has been defined as a global computer network providing a variety of information and communication facilities, consisting of interconnected networks using standardized communication protocols. The Internet has become one of the most popular media and adolescents worldwide utilize it. It is a global network connecting millions of computers. The rapid increase in Internet use in recent years has resulted in both benefits and disadvantages. A number of studies have been done which investigate the negative use of the Internet. While many believed the term addiction should be applied only to cases involving the ingestion of a drug (Rachlin, 1990; Walker, 1989), defining addiction has moved beyond this to include a number of behaviors such as compulsive gambling (Griffiths, 1990), video game playing (Keepers, 1990), overeating (Lesuire \& Bloome, 1993), exercise (Morgan, 1979), love relationships (Peele and Brody, 1975) and television viewing (Winn, 1983). As a result of these studies, the term 'Internet Addiction' has emerged.

Internet addiction is characterized by excessive or poorly controlled preoccupations, urges or behaviors regarding Internet use that lead to impairment and distress. It was not until the early 1990s that reports began to appear in the medical and psychological literature for what Griffiths called a 'technology addiction', described as a 'non-chemical addiction involving human-machine interaction'. The earliest reports on Internet addiction date back to the 1970s when scientists and academics began to express their concern with the overuse of computers. The concept of Internet addiction was first introduced by Young

\footnotetext{
${ }^{1}$ Department of Psychology, CHRIST University

${ }^{2}$ Department of Psychology, CHRISTUNIVERSITY BANGALORE

*Corresponding Author

(C) 2015 I H Sanghvi, U Rai; licensee IJIP. This is an Open Access Research distributed under the terms of the Creative Commons Attribution License (http://creativecommons.org/licenses/by/2.0), which permits unrestricted use, distribution, and reproduction in any Medium, provided the original work is properly cited.
} 


\section{Internet Addiction and its relationship with Emotional Intelligence and Perceived Stress experienced by Young Adults}

(1996). According to Young et al., Internet addiction is a broad term covering a wide variety of behaviors and impulse control behaviors. The five subtypes of Internet addiction are cyber sexual addiction, cyberrelational addiction, net compulsions, information overload and computer addiction. As the definition of Internet addiction is still under debate, there is currently no listing for the disorder in the Diagnostic and Statistical Manual of Mental Disorders-Fourth Edition (DSM-IV; American Psychiatric Association, 1995).

Some investigators have linked Internet addiction to addictive disorders, Obsessive-Compulsive Disorder (OCD) and Impulse Control Disorders (ICD). Internet addiction is best considered a disorder of impulse control because it is characterized by the failure to resist one's impulses to engage in a particular behavior despite serious personal consequences, and are considered pleasurable and are seldom resisted. Studies suggest that individuals addicted to the Internet meet the criteria for Axis-I and Axis-II disorders; mood, anxiety, substance use and ICDs. Until the Internet addiction achieves recognition as a disorder, it would be best to classify it under Axis- I 'Impulse Control Disorder not otherwise specified'. Various scales have been developed to assess Internet addiction. One of the most reliable and valid being the Internet Addiction Test created by Young.

No particular theory explains the cause of Internet addiction. The a etiology is multifactorial. However, stress has been laid on Cognitive-Behavioral theory and Social Skills deficit theory to explain the cause of Internet addiction. Cognitive behavioral therapy (CBT) is a psychotherapeutic approach that addresses dysfunctional emotions, behaviors, and cognitions through a goal-oriented, systematic process. The cognitive behavioral theory can explain the onset and maintenance of pathological Internet use. The Social Skills Deficit theory holds the assumption that lonely and depressed individuals hold negative views of their social competence. It also assumes that computer-mediated communication interactions give people a greater flexibility in self-presentation than face-to-face communication, and one may omit or edit information they feel is negative or harmful.

Individuals addicted to the Internet may use the Internet for extended periods, isolating themselves from other social contact, and focus almost entirely on the Internet rather than broader life events. Adolescents with Internet addiction usually suffer from problems with their daily routines, school performance, family relationships and mood. The most characteristic symptom of Internet addiction is excessive 'nonessential' time spent online. This term refers to time not related to work or academic pursuits. Internet addiction has also been associated with emotional intelligence.

Emotional Intelligence involves the ability to monitor one's own and others' feelings and emotions, to discriminate among them and to use this information to guide one's thinking and actions (Salovey and Mayer, 1990). The study and measurement of emotional intelligence has its roots in the works of such psychometric pioneers such as Binet, Thorndike and Wechsler among others (Fancher, 1985). Daniel Goleman (1995) identified the five 'domains' of EQ as: knowing your emotions, managing your own emotions, motivating yourself, recognizing and understanding other people's emotions and managing relationships, i.e., managing the emotions of others. Various tests have been created to measure emotional intelligence. Of them Mayor-Salovey-Caruso Emotional Intelligence Test (MSCEIT) and the Emotional Intelligence Scale (EIS) are the most valid and reliable. Studies have showed the correlation of emotional intelligence and addictions. Healthy emotional development has been recognized as essential to an 


\section{Internet Addiction and its relationship with Emotional Intelligence and Perceived Stress experienced by Young Adults}

individual's psychological and physical well-being. In this study an attempt is made to study the effect of Internet addiction on emotional Intelligence.

Studies show that individuals addicted to the Internet experience high levels of stress and anxiety. Thus it is obvious that stress and Internet addiction are correlated. Stress has various definitions. Psychologically, it can be defined as a state of bodily or mental tension resulting from factors that tend to alter an existent equilibrium. It is a psychological and physical response of the body that occurs whenever we must adapt to changing conditions, whether those conditions be real or perceived, positive or negative. Walter Cannon, first described the effects of stress by terming it as fight or flight response. Various other theories have formulated on stress. Some of them being the general adaptation syndrome by Hans Selye, hardiness theory by Kobasa and colleagues and the social support theory. Stress has been positively correlated to Internet addiction as found by many studies. The Perceived Stress Scale (PSS) is the most widely used psychological instrument for measuring the perception of stress.

This study tries to study Internet addiction and its effect on emotional intelligence and the stress experienced by young adults of age between 17-22 years.

\section{REVIEW OF LITERATURE}

Internet addiction is a disorder that occurs worldwide, but mainly in countries where computer access and technology are widespread. The condition has attracted increasing attention in the field of Psychology and among researchers. Internet addiction is characterized by excessive or poorly controlled preoccupations, urges or behaviors regarding computer use and Internet access that lead to impairment and distress. Several things seem to be correlated with Internet addiction. Some of them are depression. anxiety, emotional intelligence, stress and loneliness. The current study focuses on Internet addiction and its affect on emotional intelligence and stress experienced by young adults. The study hypothesizes that 1) there is a correlation between Internet addiction and emotional intelligence 2) there is a correlation between Internet addiction and the stress experienced due to it by young adults (17-22years). The following five literature reviews attempt to demonstrate and support the hypothesis.

In a research article by Martha Shaw and Donald W. Black (2008) an attempt was made to define Internet addiction, ways to assess Internet addiction, its epidemiology and clinical management. Currently, there is no listing for the disorder in DSM-IV-TR. As the appropriate classification of Internet addiction has been debated, this article proved to be a stepping-stone for further research and gave a clear picture of the disorder. According to the article, some researchers have linked Internet addiction to addictive disorders, grouping it alongside alcohol and drug use disorders. Internet addiction has also been linked to Obsessive Compulsion Disorder (OCD) and Impulse Control Disorder (ICD). Young et al., described five behaviors under Internet addiction: cyber sexual addiction, cyber-relational addiction, net compulsions, information overload and computer addiction. The article also focused on the assessment on Internet Addiction. Findings suggested that the psychiatric history of the patient was to be explored because many individuals with Internet addiction meet the criteria for co-morbid psychiatric disorders such as major depression, ICD or anxiety disorder. Several screening instruments have been developed to assess Internet addiction although none as emerged as a standard one. Brenner developed the Internet Addictive Behavior Inventory (IRABI) and Young created the Internet Addiction Test (IAT). The IAT appears to be valid and reliable. The article also looks at the epidemiology of the disorder. Findings suggest that the disorder has 


\section{Internet Addiction and its relationship with Emotional Intelligence and Perceived Stress experienced by Young Adults}

an age of onset in the late 20s or early 30s (Black et al., Shapira et al.) while men and women did not differ in the amount of time spent online or problems experienced, older users reported fewer problems than younger ones. The article also throws light on the aetiology of the disorder. The cause of Internet addiction is unknown. However, according to Davis, the cognitive behavioral therapy can explain the onset and maintenance of pathological Internet use. Caplan explains the disorder using the Social Skills Deficit Theory. Neurobiological theories have also helped in explaining the disorder. Important gaps remain in the understanding of this disorder. Firstly, in the assessment of Internet addiction, there are various scales that measure the extent of Internet addiction but their reliability and validity have not been clearly determined. Secondly, the exact onset of the disorder needs to be tracked. Further research in this area can tackle these issues.

In his studies, Greenfield found that online addicts feel a sense of displacement when online, and are unable to manage central aspects of their lives due to their growing preoccupation with online use. They start to miss important deadlines at work, spend less time with their family, and slowly withdraw from their normal routines. They neglect social connections with their friends, coworkers, and with their communities, and ultimately, their lives become unmanageable because of the Internet. As the addiction grows, they become consumed with their Internet activities, preferring online games, chatting with online friends, or gambling over the Internet, gradually ignoring family and friends in exchange for solitary time in front of the computer. Subsequently, in a study conducted by the Stanford Institute for the Quantitative Study of Society, researchers found that use of the Internet could lead to loneliness and a decline in social engagement. While controversial, the researchers concluded that "the more hours people use the Internet, the less time they spend in contact with real human beings.” In a two-year longitudinal study, Kraut and his colleagues randomly selected families who were given computers and instruction on Internet use, and after a two-year follow-up, higher levels of depression and loneliness were reported by respondents who used the Internet as little as a few hours a week. In fact, the study reported that greater use of the Internet led to shrinking social support and happiness, and increase in depression and loneliness. In studies specific to addictive use of the Internet, Young found that social isolation was directly associated with compulsive Internet use in nearly $56 \%$ of 396 cases of online addicts she surveyed.

To date, only few neuroimaging studies had been performed to investigate brain structural and functional changes associated with Internet addiction disorder. In an article by Fuchun Lin, Lindi Qin and Yuan Chi, it was found that Internet addiction disorder subjects had multiple structural changes in the brain, and such changes correlated significantly with the duration of Internet addiction. Seventeen IAD subjects and sixteen healthy controls without IAD participated in this study. Whole brain voxel-wise analysis of fractional anisotropy (FA) was performed by tract-based spatial statistics (TBSS) to localize abnormal white matter regions between groups. A voxel-based morphometry (VBM) study reported decreased gray matter density in the left anterior cingulate cortex, posterior cingulate cortex, insula and lingual gyrus of Intermet addiction disorder adolescents. Dong et al. reported that IAD students had lower activation in the conflict detection stage, and showed less efficiency in information processing and lower impulse control than normal controls by recording event-related brain potentials during a Go/No-Go task. Additionally, a positron emission tomography (PET) study found that Internet game overuse shares psychological and neural mechanisms with other types of impulse control disorders and substance/non-substance-related addiction. Taken together, these findings indicate that IAD subjects are associated with structural and 


\section{Internet Addiction and its relationship with Emotional Intelligence and Perceived Stress experienced by Young Adults}

functional changes in brain regions involving in emotional processing, executive attention, decision making and cognitive control.

Studies have showed the correlation between emotional intelligence and Internet addiction. Various researches verify the relationship between EI and addictive disorders. Internet addiction, which has also been considered as one of the addictive disorders, correlates with EI. In an article by Bernadette Kun and ZsoltDemetrovics, the correlation between EI and addictions has been explored. Addicts were more vulnerable to emotion regulation problems than others. OCD shows a high correlation not only with the use of psychoactive substances (Regier et al., 1990) but with behavioral addictions also (Frost, Krause \&Steketee, 1996; Grant, Mancebo, Pinto, Eisen, \& Rasmussen, 2006; Holderness, Brooks-Gunn, \& Warren, 1994). Internet addiction is a type of behavior addiction. A very important empirical finding attempted to explore the relationship between addiction and Alexithymia. Characteristics of alexithymia are: 1) difficulty identifying feelings and distinguishing between emotions and corresponding bodily sensations 2) difficulty in describing feelings to others 3) constricted imaginal life and fantasies 4) externally oriented cognitive style (Nemiah, Freyberger, \& Sifneos, 1976). The article used the Cochrane Collaboration and proposals of Stroup and colleagues (2000) in reporting and designing the study. Various databases like PsycINFO, MEDLINE, PubMed, Web of Science and EBSCO were used for the review of literature for this article. The article published various relationships between EI and addiction problems between 1990 and May 2009. One of the findings was that of Internet addiction. Engelberg and Sjoberg conducted the study, 2004 in Sweden. The study was conducted on 41 university students and Internet addiction was assessed using the MSCEIT. A correlation was seen between the two variables. According to the article, people scoring higher on the Internet Addiction Scale (Young, 1998) performed worse in emotion-decoding tasks. Similar results appeared in a study by Parker et al., 2008. The Internet Addiction Scale (Young, 1998) negatively correlated with the Emotional Quotient Inventory Youth Version (EQ-i YV) (Bar-on \& Parker, 2000). Results showed that 'stress management' and 'adaptability' were particularly important EI components for those addicted to the Internet. Thus, this article opens the door to study the effect of Internet addiction on EI, one of the objectives of the current study.

Studies have showed the relation between EI and Internet addiction. In one another article, by VesileOktan (2011) the predictive relationship between emotion management skills and Internet addiction. Emotion management skills are an important part of EI. Emotion management skills involve verbal and spontaneous expression of emotions, the ability to manage emotions and control negative physical reactions, coping and anger management (Cecen, 2006). Researchers believe that the development of Internet addiction is associated with ability to control emotions and emotional reactions. In the present article, criteria used in defining Internet addiction are related to emotional control. They include failing to control, decrease or stop Internet use, needing to increasingly use the Internet and staying logged on longer than planned (Young, 1998). The sample of the present study comprised a total of 345 students (185 females, 160 males), approximately 19 years of age, falling in the range of 17-22 years. Simple random sampling was done. The instrument used for the study was the Emotion Management Skills Inventory (EMSI, Cecen, 2006). The inventory consists of 28 items and is a 5-point Likert type self-evaluation instrument that is used to measure emotion management skills. The Problematic Internet Usage Scale (PIUS, Ceyhan, \&Gurcan, 2007) was another instrument used for the study. The scale consists of 28 items and is used to identify the levels of positive and negative use of the Internet. Multilinear regression analysis was used to derive the results. It was seen that emotion management skills were meaningfully related to average and above average levels of Internet addiction. 


\section{Internet Addiction and its relationship with Emotional Intelligence and Perceived Stress experienced by Young Adults}

Subscales like verbal expression of emotions, controlling negative physical reactions, coping and anger management did not seem to be meaningful predictors of Internet addiction. The article states Young (1998) who says, emotions are 1 of 4 factors that trigger overuse of the Internet. This article clearly underlines the relationship between Internet addiction and EI. However, it does not speak whether the effect is unidirectional or bidirectional. That is it does not say whether Internet addiction leads to low EI or whether EI leads to Internet addiction or if the two have an equal influence on the other.

The relationship between EI and Internet addiction has been studied. Perhaps, there is also a relationship between Internet addiction and the stress experienced due to it. An article by Ahmet Akin and Murat Iskender (2011) examines the relationship between Internet addiction and anxiety, depression and stress. In this article, stress is operationalized as an emotional state of bodily or mental tension resulting from factors that tend to alter an existent equilibrium. The research was conducted on 300 university students in Turkey (96 males and 204 females), with a mean age of 21.1 years and the range being 17-24 years. The Online Cognition scale (OCS) was used to measure Internet addiction. The scale consists of 36 items on a 7-point Likert scale. It was developed by Davis, Flett and Besser (2002) to assess Internet addiction. The Depression Anxiety Stress Scale (DASS), a 42 item self-report inventory was used to assess depression, anxiety and stress. According to research conducted for the article, Internet addiction has been associated with some social and psychological variables such as, decline in the size of social circle, depression, loneliness (Kraut et al., 1998), lower self esteem and life satisfaction (Ko, Yen, Chen et al., 2005), sensation seeking (Lin \&Tsai, 2002), poor mental health (Yang, 2001; Young and Rogers, 1998) and low family functioning (Armstrong and Philips, \& Saling, 2000). All of these lead to stress in individuals. The authors of this study report that excessive Internet use can displace valuable time that people spend with family and friends, which leads to smaller social circles and higher levels of loneliness and stress. The findings of the study showed a positive relation to depression, anxiety and stress. The study also showed that Internet addiction enhances anxiety and stress. The more addicted to the Internet

an individual is, the more stress or anxiety he/she has. The article increases the understanding of the relationship between Internet addiction and stress levels experienced by individuals due to it.

In yet another article by Elisabeth Engelgerg and LennartSjoberg (2004), an investigation was done on the extent to which inter-personal skills, personality and EI are related to Internet addiction. The research was conducted on 41 students (20 women and 21 men) who were on an average 21.1 years old, range being 18-28 years. The UCLA Loneliness Scale, Work/Leisure Balance Scale, Internet Addiction Scale, the MSCEIT, Schwartz' value scale and the Big five inventory were used for the purpose of the study. The findings of the study suggested that fascination with socializing on the Internet may increase a sense of loneliness because usage becomes time-consuming and impairs normal functioning within the social and work-related realm leading to stress. The results show a very large difference in loneliness between high and low frequency users of the Internet. High users were lonelier. Results also confirmed the expectation that frequent Internet users would be lower than others on EI. The findings of this article shed some light on the extent to which Internet use is related to social adjustment.

Taken together, the results indicate that Internet addiction, EI and stress are correlated. It can be assumed from the above literature reviews that Internet addiction is a disorder that deserves an inclusion in DSM$\mathrm{V}$. Also, Internet addiction is negatively related to EI and positively related to stress. However, more studies need to be done in order to have a clear idea about this topic. 


\section{METHODOLOGY}

\section{Aim}

To study Internet addiction and its effect on emotional intelligence and stress experienced among young adults.

\section{Objective}

1. To assess the emotional intelligence of individuals who are addicted to the Internet.

2. To assess the stress experienced by individuals due to Internet addiction.

\section{Hypotheses}

1. There will be a significant correlation between Internet addiction and emotional intelligence.

2. There will be a significant correlation between individuals addicted to the Internet and the stress experienced by them due to Internet addiction.

\section{Sample Design}

Initially, a sample of 100 subjects falling in the age group of 17-22 years will be chosen using convenient sampling from Bangalore, India. The Internet Addiction Test by Young will be administered on them. From the results, those falling under the criteria of Internet addiction will be selected randomly. 30 subjects, identified as addicted to the Internet will form the final sample for the study. Their emotional intelligence and stress levels will be assessed using the Emotional Intelligence Scale (EIS) and Perceived Stress Scale (PSS).

\section{Inclusion Criteria}

- The subjects need to fall in the age between 17-22 years.

- The subjects must be able to read, write and understand English.

- The subjects should have been exposed to the Internet in the past 4 weeks.

\section{Exclusion Criteria}

- Individuals using the Internet only for work or academic purpose will not be included.

\section{Assessment tools}

1. Dr. Kimberly Young's Internet Addiction Test.

2. The Emotional Intelligence Scale (EIS)

3. The Perceived Stress Scale (PSS)

\section{Procedure of administration}

The subjects of the present study will be randomly selected by the method of convenient sampling. Initially, the sample size will be 100. The aim and the objective of the study will be explained to them. With their consent, the first test, Young's Internet Addiction Test, will be administered individually on them. After the first test, 30 subjects who have been identified as addicted to the Internet, from the results will form the final sample of the study. Assessment of their emotional intelligence will be done using the Emotional Intelligence Scale (EIS). Their stress levels will be assessed using and the Perceived Stress Scale (PSS). Correlational analysis will be used to obtain the final results of the study. 


\section{Ethical Considerations}

- The subjects would be clearly informed about the aim and the objective of the study.

- The consent of the subjects would be taken for the participation in the study.

- Confidentiality of the subject will be maintained at all times.

- The subjects would be advised to give their initials while administering the test so that confidentiality can be maintained.

- The subject would be free to opt out of the study if they find it uncomfortable.

\section{RESULTS AND DISCUSSION}

The aim of the study was to assess Internet addiction and its effect on emotional intelligence and perceived stress experienced among young adults. The study was conducted on a sample population of 30 individuals, both male and female, aged between 16-22 years with a mean age of 19.83 years, living in Bangalore. Three scales were used for the purpose of the study, namely, the Emotional Intelligence Scale, the Perceived Stress Scale and the Internet Addiction Test.

The Internet addiction Test, developed by Young is a 20-item questionnaire with a 5-point likert scale. The results are interpreted on the basis of 4 main categories. The normal range is for scores between 0-30, mild range for scores between 31-49 moderate range for scores between 50-79 and severe range for scores between 80-100. The sample population falls in the mild and moderate range. Scores of the sample population on Internet addiction range from 32-64. There are 20 participants with mild Internet addiction $(\mathrm{M}=39.25, \mathrm{SD}=5.379)$. There are 10 participants with moderate Internet addiction $(\mathrm{M}=55.7, \mathrm{SD}=4.715)$. There were no participants with severe Internet addiction.

Table 1, The mean scores and standard deviation of the sample population on mild and moderate Internet addiction

\begin{tabular}{llllll} 
& N & Minimum & Maximum & Mean & Std. Deviation \\
mild & 20 & 32 & 48 & 39.25 & 5.379 \\
moderate & 10 & 50 & 64 & 55.70 & 4.715 \\
\hline
\end{tabular}


Figure 1. The mean Internet addiction of the sample population on the two categories of Internet addiction, mild and moderate

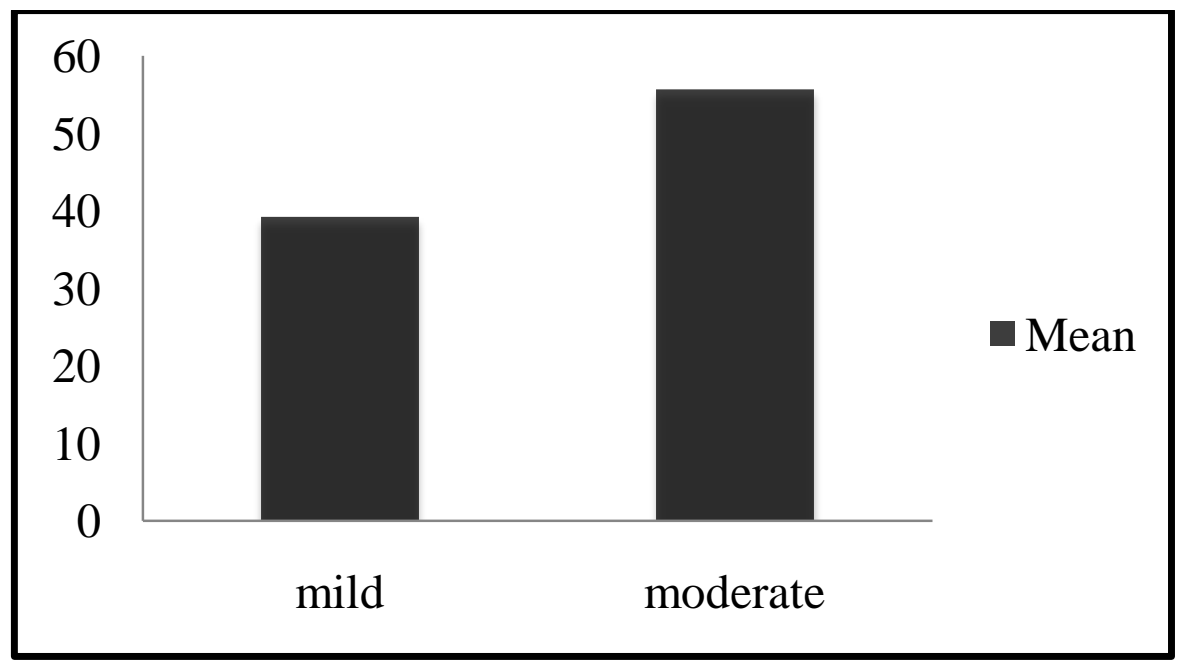

An adapted version of the Emotional Intelligence scale developed by Anukool Hyde, Sanjyot Pethe and Upindher Dhar was used. The scale consists of 34 items. The results are interpreted based upon 3 categories. Scores of 85 and above are interpreted as high. Those in the range of 52-84 are interpreted as normal and scores of 51 and below are interpreted as low. All the participants of the sample population were found to have a high emotional intelligence scores assessed by the Emotional Intelligence Scale $(\mathrm{M}=131.9, \mathrm{SD}=11.028)$.

The Perceived Stress Scale is a 10-item questionnaire. The results are interpreted based upon 5 categories. The scores of 0-7 are interpreted to very low, 8-11 low, 12-15 average, 16-20 high and 20 and above very high. The sample population belongs to average, high and very high perceived stress levels. The lowest score of the sample population is 13 and the highest is 35. A sample of five participants havean average level of perceived stress level $(\mathrm{M}=14, \mathrm{SD}=0.707)$ A sample of 13 participants belongs to a high level of perceived stress $(\mathrm{M}=18.08, \mathrm{SD}=1.605)$. A sample of 12 participants belongs to a very high level of perceived stress $(M=25.17, S D=3.904)$. None of the participants have a low or very low perceived stress levels.

Table 2, The mean scores and standard deviation of the sample population on average, high and very high levels of perceived stress

\begin{tabular}{llllll}
\hline & N & Minimum & Maximum & Mean & Std. Deviation \\
\hline average & 5 & 13 & 15 & 14.00 & .707 \\
high & 13 & 16 & 20 & 18.08 & 1.605 \\
very high & 12 & 21 & 35 & 25.17 & 3.904 \\
\hline
\end{tabular}


Figure 2. The mean perceived stress of the sample population on the 3 different categories of perceived stress, average, high and very high

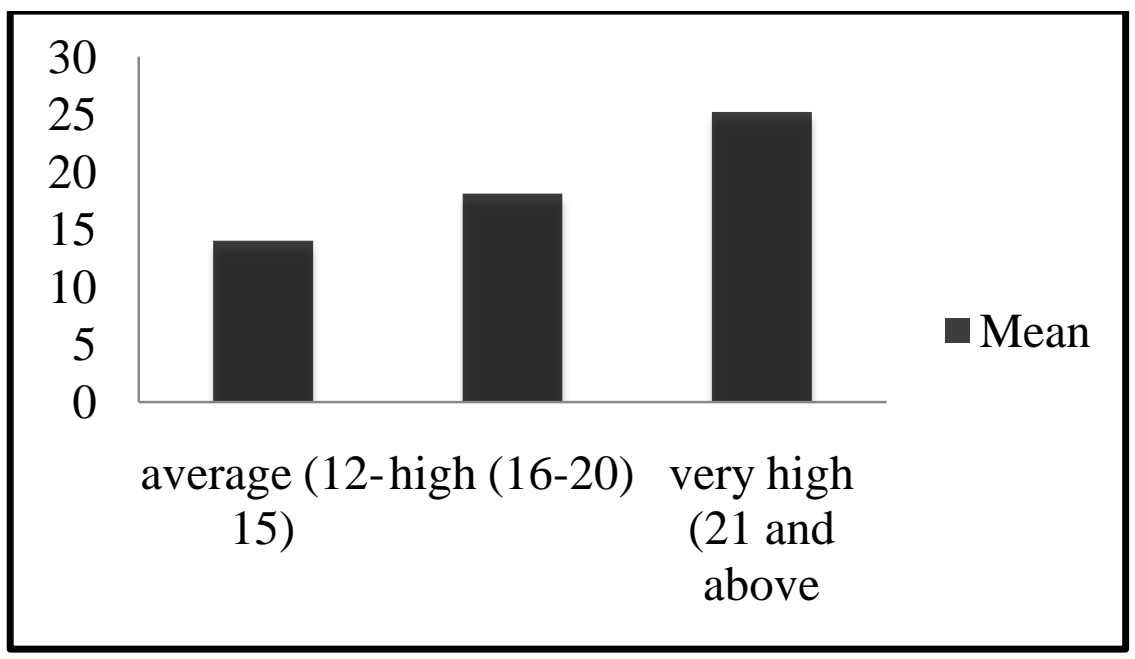

Karl Pearson Product Moment Correlation, a statistical tool was employed to study the interaction between Internet addiction and emotional intelligence and Internet addiction and perceived stress.

Table 3 The correlation of the 2 dependent variables, emotional intelligence and perceived stress with the independent variable, Internet addiction:

\begin{tabular}{lllll}
\hline & & IA Scores & EI Scores & PSS Scores \\
\hline \multirow{3}{*}{ IA Scores } & Pearson Correlation & 1 & .133 & .203 \\
& Sig. (2-tailed) & & .482 & .283 \\
& $\mathrm{~N}$ & 30 & 30 & 30 \\
& Pearson Correlation & .133 & 1 & -.202 \\
EI Scores & Sig. (2-tailed) & .482 & & .284 \\
& $\mathrm{~N}$ & 30 & 30 & 30 \\
& Pearson Correlation & .203 & -.202 & 1 \\
& Sig. (2-tailed) & .283 & .284 & \\
& $\mathrm{~N}$ & 30 & 30 & 30 \\
\hline
\end{tabular}




\section{Internet Addiction and its relationship with Emotional Intelligence and Perceived Stress experienced by Young Adults}

By referring to the above table, it is seen that for a sample population of 30, the correlation between Internet addiction and emotional intelligence is positive $(\mathrm{r}=.133)$. This indicates the correlation is not significant at both the levels. The correlation between Internet addiction and perceived stress is positive $(\mathrm{r}=.203)$. This indicates the correlation is not significant at both the levels. The table also shows the correlation between emotional intelligence and perceived stress. The correlation is a negative ( $\mathrm{r}=-.202)$. This correlation is not significant at both the levels. Inferring from the above results, it is found that the null hypothesis that states 'there will be no significant correlation between Internet addiction and emotional intelligence' and 'there will be no significant correlation between individuals addicted to the Internet and the perceived stress experienced by them due to Internet addiction' has been accepted.

Works related to Internet addiction, emotional intelligence and perceived stress have been reviewed. There are a limited number of articles related to behavioral addictions like Internet addiction, impulsive buying and problem gambling. Internet addiction has grown to become an interesting topic of study in the past one decade. The disorder has not been included in DSM-IV-TR and there is little agreement regarding its definition. Internet addiction is characterized by excessive or poorly controlled preoccupations, urges or behaviors regarding Internet use that lead to impairment and distress. Research has not clearly been able to identify whether the disorder is more prevalent in males or females, however literature suggests a male preponderance (Black D.W. and Shaw M., 1999). Studies done by Black et al. in 1999, and Shapira N., Goldsmith T. and Keck P., in 2000 suggests that there is a possible comorbidity between Internet addiction and other psychiatric disorders, mainly mood disorders. In a research done by Kraut R., Patterson M., and Landmark V. in the year 1998 revealed that an increased use of the Internet had been associated with depression, decrease in social activities and loneliness. Black et al. also suggested the prevalence of personality disorders in individuals addicted to the Internet. In the same study, most of the participants met the criteria for borderline personality, narcissistic and antisocial personality disorder.

In the present study, the participants that were mildly and moderately addicted to the Internet were found to have a high emotional intelligence. However, no significant relationship was found between Internet addiction and emotional intelligence. In the light of these findings, a previous research done by Engel berg E. and Sjoberg L. in the year 2004 suggests that individuals addicted to the Internet have a low emotional intelligence score as assessed by Emotional Quotient Inventory: Youth Version questionnaire. They suggested that frequent users of the Internet tend to lack the emotional competence characteristic of high EI. Their findings revealed two main components of emotional intelligence that play a key role in addictions, namely regulation of emotions, decoding and differentiation of emotions. However, decoding of emotional states was less prominent in cases of behavioral addictions like Internet addiction. Whereas, a weaker ability of emotion regulation was observed in individuals addicted to the Internet. Engel berg E. and Sjoberg L. also made an assumption that in order to conform to the demands of social life and adjust to the social context, an ability to understand emotions as perceived by others is useful. The difference in the findings of the present study and those done previously could be attributed to the fact that the participants of the present study were only mildly and moderately addicted to the Internet as assessed by Young's Internet Addiction Test. There could be a significant correlation between Internet addiction and emotional intelligence for individuals who are severely addicted to the Internet.

The present study also focuses on the relationship between Internet addiction and perceived stress. In the present study, individuals mildly and moderately addicted to the Internet have been found to have 


\section{Internet Addiction and its relationship with Emotional Intelligence and Perceived Stress experienced by Young Adults}

average, high and very high levels of perceived stress as assessed by the Perceived Stress Scale. This could lead us to believe that the more an individual is addicted to the Internet, the higher stress levels he perceives. However, no significant relationship was found between Internet addiction and perceived stress. Minimal literature is available on the effect of Internet addiction and perceived stress. In a research done by Nie NH. and Erbring L., in the year 2007, it was found that excessive Internet use can displace valuable time that people spend with family and friends, which leads to smaller social circles and higher levels of loneliness and stress. Since the greater use of the internet is associated with some social and psychological maladaptive variables such as, declines in the size of social circle, loneliness (Yang, 2001), lower self-esteem and life satisfaction (Ko, Yen, Chen et al., 2005), sensation seeking (Lin \& Tsai, 2002), poor mental health (Yang, 2001; Young \& Rogers, 1998), and low family function (Armstrong et al., 2000), Internet addiction may enhance stress. On basis of these findings, there could be a possible correlation between the individuals severely addicted to the Internet and their perceived stress levels.

\section{SUMMARY AND CONCLUSIONS}

The present study aimed at examining the relationship of Internet addiction with emotional intelligence and perceived stress as experienced by young adults in Bangalore.

The objective of the study was to assess the emotional intelligence of individuals who are addicted to the Internet and assess the perceived stress experienced by individuals due to Internet addiction.

\section{Two null hypotheses were generated:}

1. There will be no significant correlation between Internet addiction and emotional intelligence.

2. There will be no significant correlation between individuals addicted to the Internet and the stress perceived by them due to Internet addiction.

A sample of 30 individuals, ranging between the age of 16-22 years with a mean age of 19.83 years were used for the purpose of the study. The data for the same was collected using three questionnaires; Young's Internet Addiction Test, the Emotional Intelligence Scale and the Perceived Stress Scale.

Correlational analysis was employed to the study the relationship of Internet addiction with emotional intelligence and perceived stress. It was found that there was no significant correlation between Internet addiction and emotional intelligence and between Internet addiction and perceived stress. Thus, the null hypotheses have been accepted.

\section{LIMITATIONS}

Important gaps remain in the understanding of the current research topic. The study was based on a small sample. A bigger sample would provide a better picture on the topic. Participants were only mildly and moderately addicted to the Internet. No participants were found with severe Internet addiction. Severe Internet addiction will possibly show a different correlation with emotional intelligence and perceived stress. Also, a control group could be used to compare the effect of emotional intelligence and perceived stress levels of individuals addicted to the Internet to those not addicted to the Internet. The scale used to measure Internet Addiction has not been globally accepted. This is a major drawback of the study. 


\section{IMPLICATIONS}

The present study demonstrated that there is no significant correlation between mild and moderate Internet addiction and emotional intelligence as well as perceived stress. The study adds to the existing literature on Internet addiction, emotional intelligence and perceived stress in the Indian context. The findings of the study reveal that Internet addiction, unless severe, does not have negative effects on an individual's lifestyle. The study can provide a foundation of which other researchers may conduct further studies.

\section{SCOPE FOR FURTHER RESEARCH}

This research has opened pathways for further research on Internet addiction in the Indian context. A larger sample population from the different metropolitan cities of India can provide a better understanding of the subject and its prevalence in India. Gender differences with regard to Internet addiction can be studied. Further research can also explore the relationship between emotional intelligence and perceived stress.

American Psychiatric Association, (1995).Diagnostic and Statistical Manual of Mental Disorders. $4^{\text {th }}$ ed., text rev. Washington, DC.

Akin, A., Iskender, M. (2011). Internet addiction and depression, anxiety and stress. International Online Journal Of Educational Sciences, 3(1), 138-148.

Asendorpf, J.B. (1998). Personality effects on social relationships. Journal of Personality and Social Psychology, 74, 1531-1544.

Black, W.D., Shaw, M. (2008). Internet addiction definition, assessment, epidemiology and clinical management.CNS Drugs, 22(5), 353-365.

Davis, R.A. (2001). A cognitive-behavioral model of pathological internet use. Computers in Human Behavior, 17(2), 187-195.

Dr. Young, K. (1996). Internet addiction: the emergence of a new clinical disorder. Paper presented at the $104^{\text {th }}$ annual meeting of the American Psychological Association, August 11, 1996. Toronto, Canada.

Dr. Young. K. (1999). Internet addiction: symptoms, evaluation and treatment. Innovations in Clinical Practice, vol 17.

Engelberg, E., Sjoberg, L. (2004). Internet use, social skills and adjustment. Cyber Psychology \& Behavior, vol 7 (1).

Griffiths, M. (1995). Technological addictions. Clinical Psychology Forum, 76, 14-19.

Kun, B., Demetrovics, Z. (2010). Emotional intelligence and addictions: a systematic review. Substance Use \& Misuse, 45, 1131-1160.

Oktan, V. (2011). The predictive relationship between emotion management skills and internet addiction. Social Behavior and Personality, 39(10), 1425-1430.

Weinstein, A., Lejoyeux, M. (2010). Internet addiction or excessive internet use. The American Journalof Drug and Alcohol Abuse, 36, 277-283. 